Carol Di Perri, MD

Stefano Bastianello, MD

Andreas J. Bartsch, MD, $\mathrm{PhD}$

Caterina Pistarini, MD

Giorgio Maggioni, MD

Lorenzo Magrassi, MD

Roberto Imberti, MD

Anna Pichiecchio, MD

Paolo Vitali, MD

Steven Laureys, MD,

$\mathrm{PhD}$

Francesco Di Salle, MD

Correspondence to

Prof. Di Salle:

francesco.disalle@

maastrichtuniversity.nl

Supplemental data at www.neurology.org

\title{
Limbic hyperconnectivity in the vegetative
}

state

\section{ABSTRACT}

Objective: To investigate functional connectivity between the default mode network (DMN) and other networks in disorders of consciousness.

Methods: We analyzed MRI data from 11 patients in a vegetative state and 7 patients in a minimally conscious state along with age- and sex-matched healthy control subjects. MRI data analysis included nonlinear spatial normalization to compensate for disease-related anatomical distortions. We studied brain connectivity data from resting-state MRI temporal series, combining noninferential (independent component analysis) and inferential (seed-based general linear model) methods.

Results: In DMN hypoconnectivity conditions, a patient's DMN functional connectivity shifts and paradoxically increases in limbic structures, including the orbitofrontal cortex, insula, hypothalamus, and the ventral tegmental area.

Conclusions: Concurrently with DMN hypoconnectivity, we report limbic hyperconnectivity in patients in vegetative and minimally conscious states. This hyperconnectivity may reflect the persistent engagement of residual neural activity in self-reinforcing neural loops, which, in turn, could disrupt normal patterns of connectivity. Neurology ${ }^{\circledR} 2013 ; 81: 1417-1424$

\section{GLOSSARY}

ACC = anterior cingulate cortex; $\mathbf{D M N}=$ default mode network; $\mathbf{G L M}=$ general linear model; ICA $=$ independent component analysis; $\mathbf{M C S}=$ minimally conscious state; $\mathbf{P C C}=$ posterior cingulate cortex; $\mathbf{R O I}=$ region of interest; $\mathbf{V S}=$ vegetative state; VTA $=$ ventral tegmental area.

Patients in a vegetative state (VS) (also called "unresponsive wakefulness syndrome"1) exhibit apparent dissociation between wakefulness and awareness, the 2 cardinal elements of consciousness. ${ }^{2}$ Onset of a VS, in which awareness is negatively affected, typically follows a coma and can be chronic and may evolve into a minimally conscious state (MCS). ${ }^{3}$ The current literature assumes a "passive" model of unawareness in both the VS and MCS, which is associated with widespread cerebral connectivity loss.

In severe brain injury and postcomatose states, the default mode network (DMN), a major frontoparietal connectivity network of the resting brain connecting anterior and posterior cingulate with parietal and hippocampal regions, shows a breakdown of connectivity depending on the level of consciousness. ${ }^{4}$ Similar findings were found in healthy controls during altered states of consciousness such as anesthesia and sleep. ${ }^{5}$

The $\mathrm{DMN}^{6}$ has been proposed as a correlate of the baseline cognitive state of a subject, and its link to memory and executive functions in normal and pathologic conditions suggests profound implications for consciousness. ${ }^{7}$

To date, interplay between the DMN and other brain networks has not been well explored in disorders of consciousness. We hypothesized that neural connectivity measured by resting-state

\footnotetext{
From the Department of Neuroradiology (C.D.P., A.P., P.V.), National Neurological Institute C. Mondino, Pavia; Department of Neuroradiology (S.B.), Neurological Institute C. Mondino, University of Pavia, Italy; Coma Science Group (C.D.P., S.L.), Cyclotron Research Centre and Neurology Department, University and University Hospital of Liège, Belgium; Department of Cognitive Neuroscience (C.D.P., F.D.S.), Faculty of Psychology and Neuroscience, Maastricht University, the Netherlands; Department of Neuroradiology (A.J.B.), University of Heidelberg, Germany; Functional MRI of the Brain (A.J.B.), University of Oxford, UK; Neurorehabilitation Unit (C.P., G.M.), IRCCS, S. Maugeri Foundation, Via Maugeri, Pavia, Italy; Department of Surgical Sciences, University of Pavia (L.M.), Department of Anesthesiology and Critical Care Medicine (R.I.), Fondazione IRCCS, Policlinico S. Matteo, Pavia; University of Salerno (F.D.S.), Medical Faculty; and Fondazione Eugenio Medea (F.D.S.), Lecco, Italy.

Go to Neurology.org for full disclosures. Funding information and disclosures deemed relevant by the authors, if any, are provided at the end of the article.
} 
fMRI, which seems to be disengaged from the DMN in consciousness disorders, may establish or intensify functional connections with neural structures external to the DMN, possibly resulting in specific pathophysiologic consequences. Using resting-state MRI, we investigated the possibility that disorders of consciousness (VS and MCS) might not be simply a function of DMN hypoconnectivity, but rather a more complex, dysfunctional brain connectivity architecture.

METHODS Subjects. We studied a convenience sample of 11 patients in VS ( 6 males; mean age 56 years, range $23-80$ years; mean duration of disease 20 months, range 4-84 months) and 7 patients in MCS ( 6 males, mean age 36 years, range $17-56$ years; mean duration of disease 13 months, range 1-24 months).

In this study, we used one independent control group $(\mathrm{n}=12$; 5 males, mean age 48 years) to identify the seed coordinates for subsequent seed-based analyses. Another group of controls $(\mathrm{n}=$ 18 ) was then split into 2 sets of age- and sex-matched groups for the seed-based analysis: 11 controls (6 males; mean age 54 years, range 24-69 years) were compared with the 11 patients in VS, and 7 controls ( 5 males; mean age 37 years, range $23-70$ years) were compared with the 7 patients in MCS.

For clinical and demographic characteristics of study participants, see table e-1 on the Neurology ${ }^{\circledR}$ Web site at www. neurology.org.

Standard protocol approvals, registrations, and patient consents. The ethical committees of the C. Mondino Institute of Pavia approved the study. We obtained written informed consent from the legal surrogates of VS and MCS patients, or from participants themselves in the case of healthy volunteers.

Image acquisition. All subjects underwent MRI in a $1.5 \mathrm{~T} \mathrm{Phi-}$ lips Intera Gyroscan 8-channel scanner (Royal Philips Electronics Inc., Eindhoven, the Netherlands). We acquired functional time series lasting 7.5 minutes using T2-weighted echo-planar imaging (echo time $=60$ milliseconds; repetition time $=3$ seconds; field of view $=25.6 \times 25.6 \mathrm{~cm}$ with an in-plane matrix of $112 \times 112$ leading to an in-plane resolution of $2.28 \times 2.28 \mathrm{~mm}$, and 26 4-mm-thick slices). Anatomical analysis included 3D, highresolution, T1-weighted images (echo time/repetition time $=$ 4.6/25 milliseconds), fast gradient echo (fast field echo) acquisition with field of view of $25.6 \times 19.2 \mathrm{~cm}, 256 \times 192$ matrix, for an in-plane resolution of $1 \times 1 \mathrm{~mm}$.

Data analysis. Spatial normalization of scans. Given the high level of atrophy exhibited by some of the study patients, to ensure reliable intersubject alignments, we created a T1-3D template with nonlinear registration in the midspace of the template.

Creation of a midspace template. We created a custom $2 \times$ $2 \times 2 \mathrm{~mm}$ template that preserved the average brain geometry and size by applying the inverse of the mid-transform to the MNI 152 standard space template and acquired the mid-transform from full affine transformations of individual structural T1-weighted scans of participating subjects to MNI 152 space using FLIRT (version $5.5),{ }^{8}$ part of the FSL software package (release 4.1.8; www. fmrib.ox.ac.uk/fsl). ${ }^{9}$

Nonlinear registration of midspace template space. We registered each study participant to the custom midspace template ${ }^{10}$ using linear and nonlinear transformations and used FLIRT for within-subject registration of the functional echo-planar imaging data to the corresponding high-resolution structural data. We then performed cross-subject registration to the custom midspace template space using high-resolution structural data, followed by FNIRT (build 418) nonlinear registration (also part of FSL).

Preprocessing. We analyzed data using BrainVoyager QX software (Brain Innovation, Maastricht, the Netherlands) and inhouse software implemented in MATLAB (The MathWorks, Natick, MA); we aligned spatially normalized anatomical images in standardized 3D Talairach space.

For preprocessing, motion correction was applied to the functional data sets before smoothing. We used spatial smoothing for functional images using a Gaussian filter with full width at half maximum of $6 \mathrm{~mm}$ and then $>0.004 \mathrm{~Hz}$ for temporal filtering with a high-pass filter. We then aligned functional images to anatomical images and resampled to $3 \times 3 \times 3 \mathrm{~mm}^{3}$ in the custom midspace template in standardized 3D Talairach space.

Functional connectivity analysis. We used a combination of noninferential and inferential methods to estimate brain functional connectivity in the resting state, first with self-organized grouping independent component analysis (ICA ${ }^{11}$ to decompose individual time series into 25 spatial components (with associated temporal profiles). On the basis of self-organized grouping ICA results, we then conducted seed region-of-interest (ROI) correlation analyses on specific nodes of the resting-state brain activity. Because the core purpose of ICA to maximize spatial independence among connectivity components would limit ICA efficacy if a given spatial location were connected with 2 or more networks, we explored this hypothesis using seed-based analysis of DMN nodes to verify whether DMN nodes were also connected to brain structures beyond the DMN.

Independent component analysis. We performed singlesubject and group-level ICAs on the functional time series and extracted estimated independent components using the plug-in extensions for BrainVoyager QX software implementation of the fast ICA algorithm ${ }^{12}$ and the self-organizing group-level ICA algorithm. ${ }^{11}$ For each subject, 25 independent components were extracted and scaled to spatial $z$ scores. First, all individual component maps from all subjects were hierarchically "clustered" in subject space using the self-organizing group-level ICA algorithm, resulting in unique assignment of all 25 individual independent components to one of 25 "clusters" of independent components. We then synthesized group-level statistical maps by modeling variance of the individual component values both within subjects (across clusters) and between subjects (within clusters) at each voxel using 2-factor analysis of variance ${ }^{13}$ with no prior masking of the brain.

From the 2-way analysis of variance, we obtained 25 singlegroup maps, which we used for a group-independent selection of the DMN cluster. The DMN component map representing the DMN was selected visually as the unique cluster generating the typical resting-state pattern coactivating the anterior and posterior cingulate and, bilaterally, the inferior parietal cortex, as the group average spatial pattern. ${ }^{14}$ Then, for the DMN cluster, we generated 1 - and 2-group contrast t-maps expressing the voxelwise statistical significance of the within- and between-group effects in a whole-brain random effects analysis of the ICA components (corrected significance level, false discovery rate $=0.05$ ).

Generation of seed ROIs. We used the following regions as seed ROIs based on Talairach peak coordinates in the main DMN nodes, as per ICA mapping of a different group of 12 healthy controls: anterior cingulate cortex (ACC), posterior cingulate cortex (PCC), right intraparietal cortex, left intraparietal cortex, right hippocampus, and left hippocampus (table e-2). Based on these 
coordinates, we generated $216 \mathrm{~mm}^{3}$ seed ROIs and hence 6 general linear model (GLM) maps (see next paragraph) in the VS and MCS patient groups. In addition, to investigate the functional connectivity of the dopaminergic nucleus ventral tegmental area (VTA), we extracted the VTA location from hippocampal ROI-based connectivity maps comparing patients and controls. To distinguish VTA connectivity in VS and MCS patients compared with controls and to avoid circularity, ${ }^{15}$ we used VTA coordinates extracted from the comparison between VS patients and controls to analyze connectivity in MCS patients, and vice versa. We visually checked all seed ROIs for each subject to ensure anatomical consistency.

Calculation of seed ROI-based functional connectivity. For each participant, we synthesized correlation maps for each seed ROI respectively using a voxel-wise, multiple-regression approach. Regressors included motion parameters, the average time course in white matter, and the average time course in CSF (as nuisance signals), the latter 2 calculated from white matter and CSF masks. These nuisance signals were used to account for fluctuations unlikely to be relevant to neuronal activity. ${ }^{16} \mathrm{We}$ then converted resultant $t$-score maps of seed ROIs to $z$-score maps hereafter referred to as "correlation maps." Masks of white matter and CSF were determined according to anatomical boundaries from each participant's high-resolution structural images using BrainVoyager QX.
Generation of functional connectivity maps (GLM maps). We performed group analyses of correlation maps for each seed ROI using BrainVoyager QX, voxel-wise 1-sample $t$ tests, and 2-sample $t$ tests to compare seed-based functional connectivity within and between groups. For 1 -sample $t$ tests, we analyzed correlation maps for each participant in the group (patients or controls, respectively, in Talairach space) and averaged $z$ scores at each voxel across all participants in the group and compared with zero. For 2-sample $t$ tests, we added analysis of correlation maps from both groups and averaged $z$ scores at each voxel within each group and then compared groups. For each seed, we restricted 2-sample $t$ tests to voxels within the mask defined by logic "or" between the 2 groups' seed map, which resulted from 1-sample $t$ tests described above. We then determined clusters with significantly differing $z$ scores between 2 groups using random effects analysis with nonparametric Monte Carlo simulations (using BrainVoyager QX). Based on resultant seed ROIs, we generated differential GLM maps (patients vs controls) for each subgroup.

RESULTS Independent component analysis. In normal controls, we reproducibly identified the $\mathrm{DMN}$ as a set of areas encompassing PCC/precuneus, ACC/medial prefrontal cortex, temporal parietal junction, and

Figure 1 ICA DMN components and comparisons in patients and controls

A

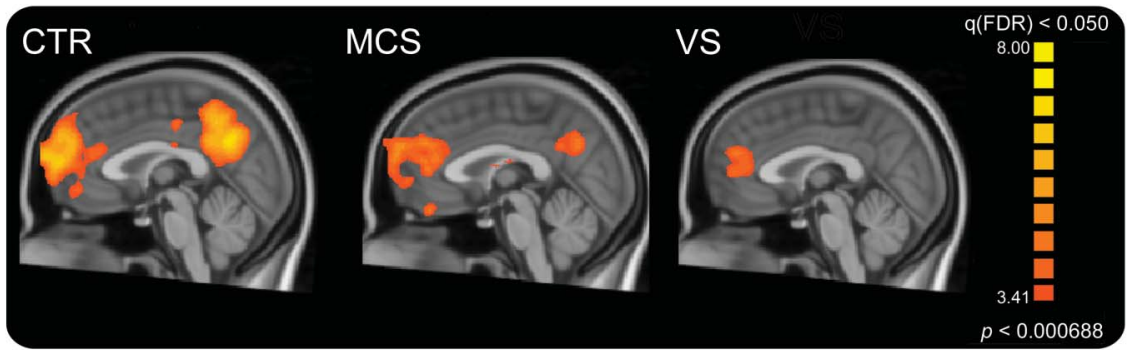

B

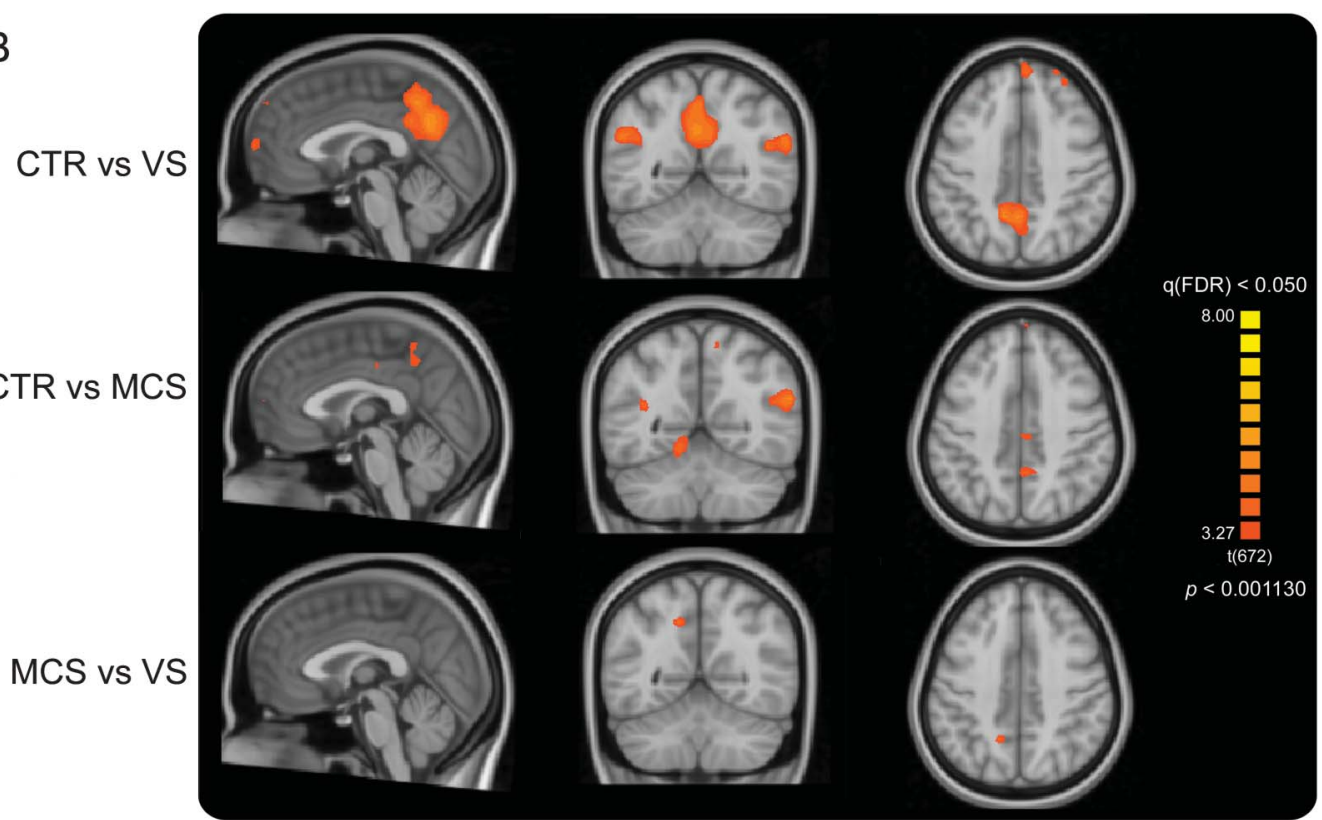

(A) Independent component analysis (ICA) default mode network (DMN) components in patients and controls (CTR). ICA DMN components in normal controls, patients in a minimally consciousness state (MCS), and patients in a vegetative state (VS). Note that the DMN spatial map is entirely represented in controls and only partially represented in VS and MCS patients. (B) ICA DMN comparison between patients and controls. Note that the DMN was less connected in patients than controls, and, among patients, more represented in MCS than VS patients. FDR = false discovery rate. 


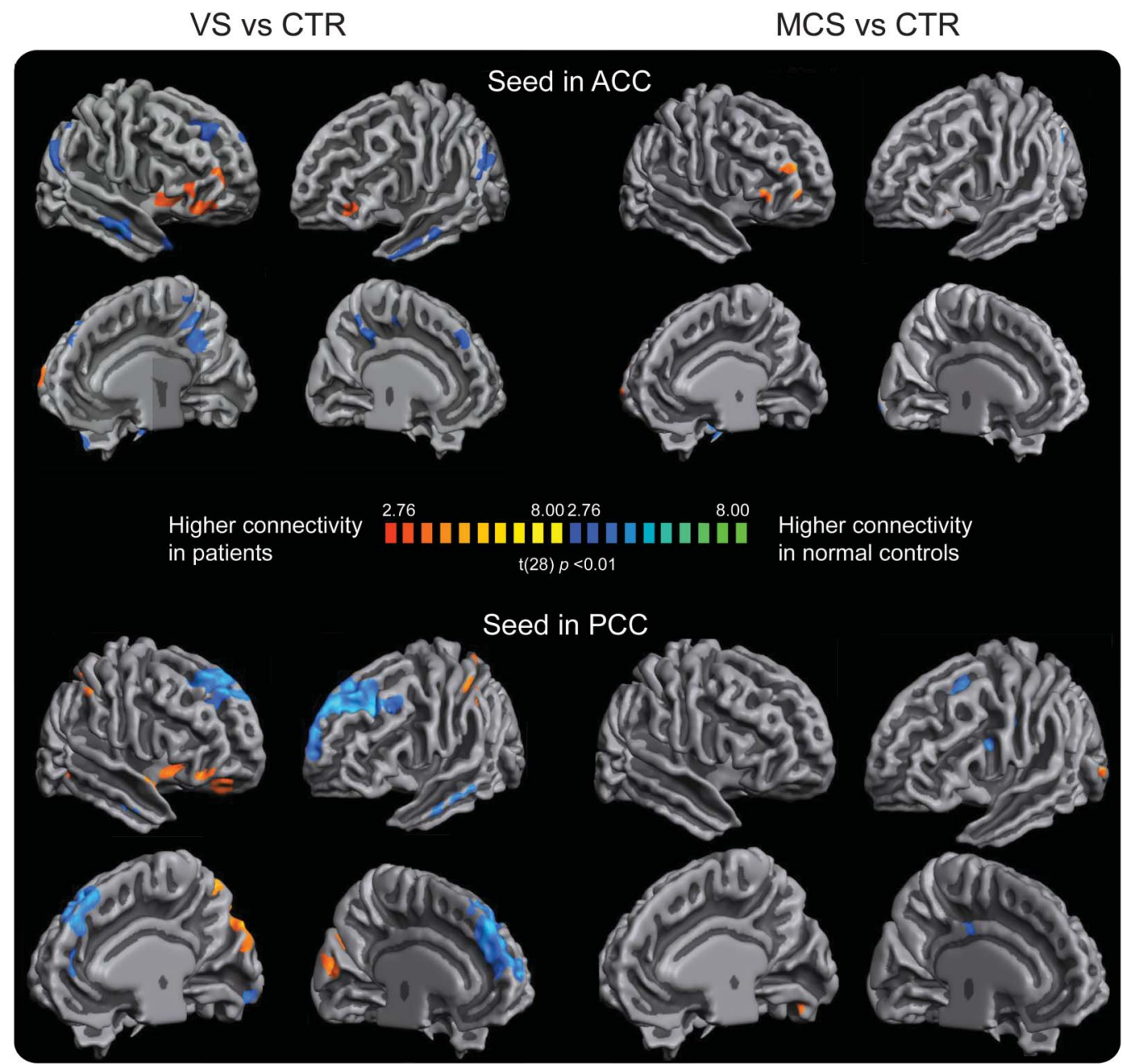

Differential general linear model maps derived from seed region-of-interest (ROI)-based correlation to the anterior cingulate cortex (ACC) and posterior cingulate cortex (PCC) in patients in a vegetative state (VS) and patients in a minimally conscious state (MCS) compared with controls (CTR), projected on mid-template surface anatomy. Results are random effects ( $p<$ 0.01 ) and cluster corrected $(p<0.05)$. Both seeds are hyperconnected to the frontoinsular cortex (in red) and hypoconnected to default mode network nodes (in blue) in VS patients compared with CTR. MCS patients show hyperconnectivity of the ACC seed ROI to the frontoinsular cortex and of the PCC seed to the occipital cortex.

hippocampus, whereas in MCS patients, areas encompassed were ACC and PCC. In VS patients, the DMN was limited to part of the ACC (figure 1A). The DMN was less connected in patients than controls, and, among patients, more represented in MCS than VS patients, especially pertaining to the PCC, while the anterior cingulate was relatively preserved (figure $1 \mathrm{~B}$ ).

GLM maps. For a summary of random effects analysis $(p<0.01)$, cluster-corrected $(p<0.05)$ results, and a comparison of VS and MCS patients, see table e-3.

We noted within-network hypoconnectivity for all DMN nodes in VS and MCS patients as compared with controls (figures 2-4 and table e-3). In contrast to previous PET studies, ${ }^{17}$ we did not observe significant hypoconnectivity between the thalamus and the DMN nodes in patients compared with controls. We also found between-network hyperconnectivity outside the DMN in VS and MCS patients compared with controls in neural components of the limbic system.

In VS patients, ACC and PCC seed ROIs were hyperconnected to insula, orbitofrontal cortex, and occipital cortex regions (figure 2); intraparietal seed ROIs were hyperconnected to insula, orbitofrontal cortex, and occipital cortex regions (figure 3); and hippocampal seed ROIs were hyperconnected to midbrain VTA, insula, and cerebellum (figure 4). In MCS patients, ACC and PCC seed ROIs were hyperconnected to insula, orbitofrontal cortex, and temporal-occipital cortex (figure 2) regions and intraparietal cortex seed ROIs were hyperconnected to temporal and occipital cortex (figure 3); hippocampus seed ROIs were hyperconnected to the parietotemporal junction. Seed ROI VTA was hyperconnected in VS patients to the hippocampal and temporal cortex 


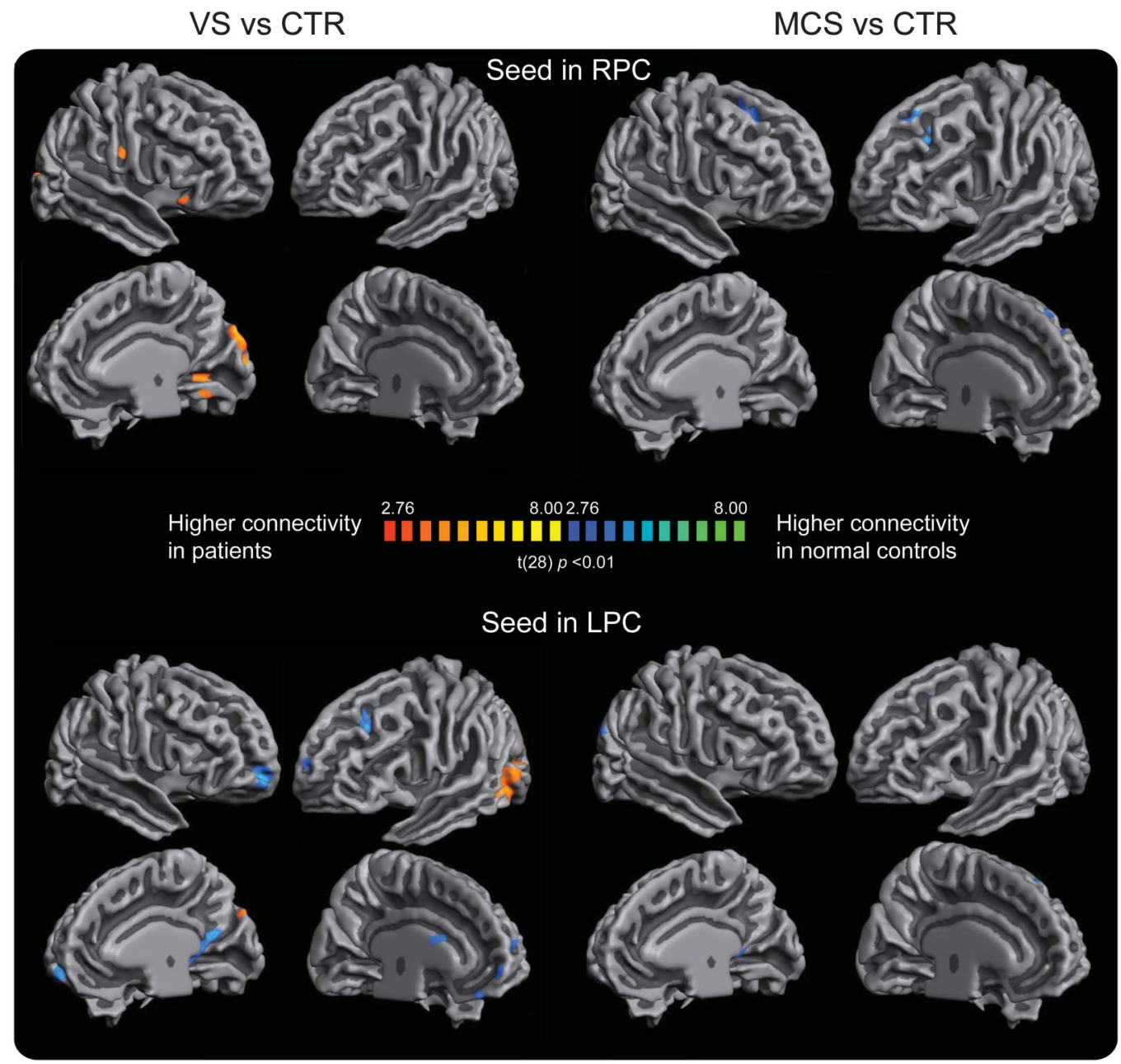

Differential general linear model maps derived from the seed region-of-interest-based correlation in the right parietal cortex (RPC) and left parietal cortex (LPC) in patients in a vegetative state (VS) and patients in a minimally conscious state (MCS) compared with controls (CTR). Results are in random effects analysis $(p<0.01)$ and cluster corrected $(p<0.05)$. Both seeds in VS patients are hyperconnected to the occipital cortex; the RPC is also hyperconnected to the orbitofrontal cortex and inferior parietal cortex, and the LPC is hypoconnected to default mode network nodes and to frontopolar and dorsolateral prefrontal cortex (DLPFC). MCS patients showed hypoconnectivity of the RPC seed to the DLPFC.

regions. However, fixed effects statistical analysis also showed hyperconnectivity between VTA and hypothalamus in both VS patients (figure 4) and MCS patients compared with controls.

DISCUSSION Our statistical analysis of brain connectivity consisting of noninferential, data-driven, and ICA showed that the DMN was correlated to the level of consciousness, similar to a recent report. ${ }^{4}$ Using seed-based analysis of DMN nodes, we also found that DMN nodes (ACC, PCC, parietal cortex, and hippocampus), while hypoconnected among themselves, were overall hyperconnected in patients to neural structures external to DMN, and more strongly in VS than in MCS patients. Hyperconnected neural circuits in patients involved mostly limbic structures, namely, insula, orbitofrontal cortex, and hypothalamus regions and included VTA nodes crucial to emotional and motivational interaction with the external environment. ${ }^{18}$

The present findings of altered connectivity do not necessarily indicate a conceptual unequivocal relationship with regional metabolism. Nevertheless, our findings are intriguingly spatially convergent with $\left[{ }^{18} \mathrm{~F}\right]$-fluorodeoxyglucose-PET studies indicating hypometabolism in frontoparietal associative networks overlapping the DMN and preserved metabolism in brainstem, hypothalamus, and basal forebrain. ${ }^{19}$ Our findings are convergent to those found in unresponsive wakefulness as seen in epilepsy, which differs from VS for being a transient state. ${ }^{20}$ During complex partial temporal epileptic seizure, similar findings of limbic hyperconnectivity have been reported, ${ }^{21}$ and marked bilateral deactivation in frontal and parietal association cortex, as already revealed in VS patients, was previously 

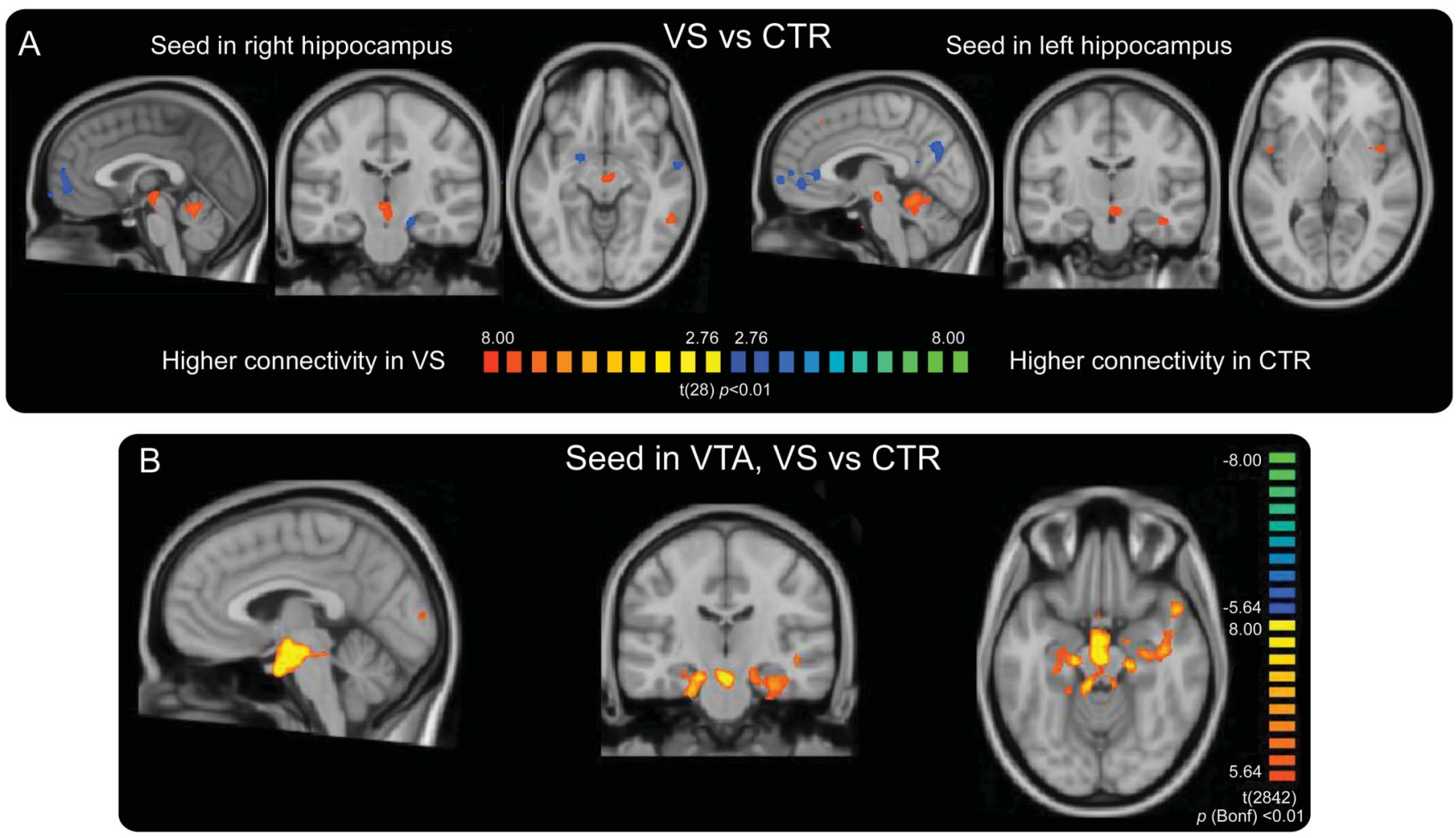

(A) Differential general lineal model (GLM) maps deriving from right and left hippocampi seed region-of-interest (ROI)-based correlation in patients in a vegetative state (VS) compared with normal controls (CTR). It shows hyperconnectivity linking the right and left hippocampi to the ventral tegmental area (VTA) and cerebellum in VS patients on triplanar anatomical slices. Images are shown in radiologic convention. Results are in random effects analysis ( $p<$ 0.01 ) and cluster corrected ( $p<0.05$ ). (B) Differential GLM maps deriving from the VTA seed ROI-based correlation in VS patients compared with normal controls. Results are in fixed effects analysis and Bonferroni corrected $(p<0.01)$. Clear hyperconnectivity links the VTA to both hippocampi and the hypothalamus in VS patients. Images are shown in radiologic convention.

described. ${ }^{2}$ Furthermore, our findings are similar to recent findings of increased functional ${ }^{22}$ and structural connectivity ${ }^{23}$ in patients with traumatic brain injury, albeit in other regions.

The observed hyperconnectivity may represent an epiphenomenon of diffusely decreased length of neural projections from/to the DMN indicating ancestral and more resistant connections between DMN nodes and limbic structures. Alternatively, increased connectivity may imply emergence of dysfunctional connections in VS and MCS, in possibly self-reinforcing loops.

ACC and PCC hyperconnectivity of the insula and orbitofrontal cortex was stronger in VS than in MCS patients. These 2 structures are important for awareness and integrate processing of multimodal sensory and interoceptive stimuli with emotional, affective, and hedonic values and reward-related reinforcers. ${ }^{24,25}$ Both hippocampi were hyperconnected to the VTA, a pivotal center of the mesocorticolimbic dopaminergic system. ${ }^{26}$ Such hyperconnectivity may represent dysregulation of a functional loop ${ }^{27}$ that is critical to interactions with the external environment ${ }^{28}$ and may affect rewardbased learning, ${ }^{29}$ novelty detection, and information storage in long-term memory. ${ }^{27}$ The mesocorticolimbic system processes motivation, emotional responses, and reward-associated behavior ${ }^{30}$ regardless of conscious awareness, ${ }^{31}$ using dopamine as the main excitatory neurotransmitter corresponding to known dopaminergic DMN sensitivity. ${ }^{32}$ In the functional link between awareness and $\mathrm{DMN},{ }^{4}$ mesocorticolimbic hyperconnectivity may have a crucial role in funneling motivational incentives into the flux of cognitive variables (e.g., memory load, stimulus rate, task engagement) that are known to modulate DMN. ${ }^{32}$

The cerebellum, which also displays increased functional connectivity with hippocampi in VS patients, does not have a direct role in mesolimbic system regulation. Nevertheless, dopamine transporter immunoreactivity studies indicate significant dopamine innervation, especially in the cerebellar vermis, ${ }^{33}$ indicating a role for the cerebellum in processing rewarding behavior as also suggested by several functional imaging reports. ${ }^{34}$

In addition, given the pivotal role of the VTA in the mesocorticolimbic circuit, ${ }^{18}$ we included this dopaminergic nucleus in the connectivity analysis. Beyond confirming increased connectivity with hippocampi, VTA showed increased connectivity to the hypothalamus, 
where it may influence neurons known to modulate reward processing and addiction ${ }^{35}$ as well as orexin(s) production, a group of wakefulness-promoting neuropeptides. ${ }^{36}$ This latter analysis did not survive random effects analysis and hence cannot be extended to the entire population.

Hyperconnectivity between the VTA and hippocampi may be explained by a hyperactive self-reinforcing loop between these nodes, mediated by the accumbens and pallidum ${ }^{27}$ and this might lead to dysregulated entry of novelty information, ${ }^{37}$ processed independently from conscious awareness. ${ }^{31}$

Herein, we illustrated a macroscopic diversion of brain functional connectivity in disordered consciousness. In VS and MCS patients, internal DMN connectivity is redirected toward deep limbic structures, inducing hyperconnectivity in an important insular/ orbitofrontal cortex workspace for awareness, propagating functional loops to alter the neural input processing of novelty/rewarding stimuli behaviors. ${ }^{27}$

This complex picture aligns with previous theories $^{38}$ explaining diffuse (extralimbic) reduction of synaptic activity in VS/MCS patients, and delineates a condition in which dysfunctional hyperconnectivity and self-reinforcing processing may impair awareness by permanently engaging critical neural resources.

The present findings are convergent with 2 further lines of evidence and with the hypothesis of a dysfunctional "mesocircuit." 38 Sporadic paradoxical enhancement of patient's neural activity after administration of CNS depressants such as zolpidem, ${ }^{39}$ may be due to interruption of limbic hyperconnectivity in the VS, making crucial neural resources available to restore normal connectivity patterns. Conversely, the efficacy of dopaminergic drugs, such as amantadine, ${ }^{39}$ may be related to functional recruitment of dopaminergic projections beyond hyperconnected dopaminergic pathways. ${ }^{26}$ The new evidence this report conveys of a limbic hyperconnectivity in patients in VS and MCS might explain the limited efficacy of the current therapeutic options and, in a far-reaching perspective, might inspire totally new treatment strategies based on the selective targeting of dysfunctional hyperconnectivity, which may be crucial to restoring consciousness. In fact, based on this new information, specific pharmacologic and/or deep brain stimulation strategies of neuromodulation may be, in principle, designed to target limbic hyperconnectivity.

\section{AUTHOR CONTRIBUTIONS}

Carol Di Perri was involved in study design, MRI acquisition, analyzed MRI data, and wrote the manuscript. Stefano Bastianello was involved in study design and data collection. Andreas Bartsch was involved in postprocessing of MRI data and study design. Caterina Pistarini and Giorgio Maggioni were involved in recruitment of patients and clinical data collection. Lorenzo Magrassi and Roberto Imberti were involved in recruitment of patients. Anna Pichiecchio was involved in study design. Paolo Vitali was involved in MRI acquisition. Steven Laureys was involved in study design and manuscript revision. Francesco Di Salle designed the study and was involved in writing the manuscript.

\section{ACKNOWLEDGMENT}

The authors thank Fabrizio Esposito, Jan Zimmermann, Mehrdad Seirafi, Joost Mulders, and Guy Poloni for technical support, and Ziggy Kennel for English editing.

\section{STUDY FUNDING}

S.L. is Research Director at the Belgian Funds for Scientific Research and is funded by the James S. McDonnell Foundation.

\section{DISCLOSURE}

The authors report no disclosures relevant to the manuscript. Go to Neurology.org for full disclosures.

Received December 28, 2012. Accepted in final form June 3, 2013.

\section{REFERENCES}

1. Laureys S, Celesia GG, Cohadon F, et al. Unresponsive wakefulness syndrome: a new name for the vegetative state or apallic syndrome. BMC Med 2010;8:68.

2. Laureys $S$. The neural correlate of (un)awareness: lessons from the vegetative state. Trends Cogn Sci 2005;9:556-559.

3. Giacino JT, Ashwal S, Childs N, et al. The minimally conscious state: definition and diagnostic criteria. Neurology 2002;58:349-353.

4. Vanhaudenhuyse A, Noirhomme Q, Tshibanda LJ, et al. Default network connectivity reflects the level of consciousness in non-communicative brain-damaged patients. Brain 2010;133:161-171.

5. Greicius MD, Kiviniemi V, Tervonen O, et al. Persistent default-mode network connectivity during light sedation. Hum Brain Mapp 2008;29:839-847.

6. Raichle ME, MacLeod AM, Snyder AZ, Powers WJ, Gusnard DA, Shulman GL. A default mode of brain function. Proc Natl Acad Sci USA 2001;98:676-682.

7. Samann PG, Wehrle R, Hoehn D, et al. Development of the brain's default mode network from wakefulness to slow wave sleep. Cereb Cortex 2011;21:2082-2093.

8. Jenkinson M, Bannister P, Brady M, Smith S. Improved optimization for the robust and accurate linear registration and motion correction of brain images. Neuroimage 2002; $17: 825-841$

9. Smith SM, Jenkinson M, Woolrich MW, et al. Advances in functional and structural MR image analysis and implementation as FSL. Neuroimage 2004;23(suppl 1): S208-S219.

10. Phillips CL, Bruno MA, Maquet P, et al. "Relevance vector machine" consciousness classifier applied to cerebral metabolism of vegetative and locked-in patients. Neuroimage 2011;56:797-808.

11. Esposito F, Scarabino T, Hyvarinen A, et al. Independent component analysis of fMRI group studies by self-organizing clustering. Neuroimage 2005;25:193-205.

12. Hyvarinen A. Fast and robust fixed-point algorithms for independent component analysis. IEEE Trans Neural Netw 1999;10:626-634.

13. Esposito F, Aragri A, Pesaresi I, et al. Independent component model of the default-mode brain function: combining individual-level and population-level analyses in resting-state fMRI. Magn Reson Imaging 2008;26:905-913.

14. Koch W, Teipel S, Mueller S, et al. Diagnostic power of default mode network resting state $\mathrm{AMRI}$ in the detection of Alzheimer's disease. Neurobiol Aging 2012;33:466-478. 
15. Kriegeskorte N, Simmons WK, Bellgowan PS, Baker CI. Circular analysis in systems neuroscience: the dangers of double dipping. Nat Neurosci 2009;12:535-540.

16. Birn RM. The role of physiological noise in resting-state functional connectivity. Neuroimage 2012;62:864-870.

17. Laureys S, Faymonville ME, Luxen A, Lamy M, Franck G, Maquet P. Restoration of thalamocortical connectivity after recovery from persistent vegetative state. Lancet 2000;355:1790-1791.

18. Fields HL, Hjelmstad GO, Margolis EB, Nicola SM. Ventral tegmental area neurons in learned appetitive behavior and positive reinforcement. Annu Rev Neurosci 2007;30:289-316.

19. Laureys S, Owen AM, Schiff ND. Brain function in coma, vegetative state, and related disorders. Lancet Neurol 2004;3:537-546.

20. Blumenfeld H. Epilepsy and the consciousness system: transient vegetative state? Neurol Clin 2011;29:801-823.

21. Zeng H, Pizarro R, Nair VA, La C, Prabhakaran V. Alterations in regional homogeneity of resting-state brain activity in mesial temporal lobe epilepsy. Epilepsia 2013;54:658-666.

22. Sharp DJ, Beckmann CF, Greenwood R, et al. Default mode network functional and structural connectivity after traumatic brain injury. Brain 2011;134:2233-2247.

23. Caeyenberghs K, Leemans A, Leunissen I, et al. Altered structural networks and executive deficits in traumatic brain injury patients. Brain Struct Funct Epub 2012 Dec 12.

24. Craig AD. How do you feel-now? The anterior insula and human awareness. Nat Rev Neurosci 2009;10:59-70.

25. Kringelbach ML. The human orbitofrontal cortex: linking reward to hedonic experience. Nat Rev Neurosci 2005;6: 691-702.

26. Adcock RA, Thangavel A, Whitfield-Gabrieli S, Knutson B, Gabrieli JD. Reward-motivated learning: mesolimbic activation precedes memory formation. Neuron 2006;50:507-517.

27. Lisman JE, Grace AA. The hippocampal-VTA loop: controlling the entry of information into long-term memory. Neuron 2005;46:703-713.
28. Hunter RG, Murakami G, Dewell S, et al. Acute stress and hippocampal histone $\mathrm{H} 3$ lysine 9 trimethylation, a retrotransposon silencing response. Proc Natl Acad Sci USA 2012;109:17657-17662.

29. Rolls ET, Xiang JZ. Reward-spatial view representations and learning in the primate hippocampus. J Neurosci 2005;25:6167-6174.

30. Wise RA. Dopamine, learning and motivation. Nat Rev Neurosci 2004;5:483-494.

31. Berridge KC, Robinson TE. Parsing reward. Trends Neurosci 2003;26:507-513.

32. Liddle EB, Hollis C, Batty MJ, et al. Task-related default mode network modulation and inhibitory control in ADHD: effects of motivation and methylphenidate. J Child Psychol Psychiatry 2011;52:761-771.

33. Hurley MJ, Mash DC, Jenner P. Markers for dopaminergic neurotransmission in the cerebellum in normal individuals and patients with Parkinson's disease examined by RT-PCR. Eur J Neurosci 2003;18:2668-2672.

34. Volkow ND, Wang GJ, Ma Y, et al. Expectation enhances the regional brain metabolic and the reinforcing effects of stimulants in cocaine abusers. J Neurosci 2003;23: 11461-11468.

35. Harris GC, Wimmer M, Aston-Jones G. A role for lateral hypothalamic orexin neurons in reward seeking. Nature 2005;437:556-559.

36. Georgescu D, Zachariou V, Barrot M, et al. Involvement of the lateral hypothalamic peptide orexin in morphine dependence and withdrawal. J Neurosci 2003;23:3106-3111.

37. Bunzeck N, Guitart-Masip M, Dolan RJ, Duzel E. Contextual novelty modulates the neural dynamics of reward anticipation. J Neurosci 2011;31:12816-12822.

38. Schiff ND. Recovery of consciousness after brain injury: a mesocircuit hypothesis. Trends Neurosci 2010;33:1-9.

39. Pistoia F, Mura E, Govoni S, Fini M, Sara M. Awakenings and awareness recovery in disorders of consciousness: is there a role for drugs? CNS Drugs 2010;24: 625-638.

\section{This Week's Neurology ${ }^{\circledR}$ Podcast}

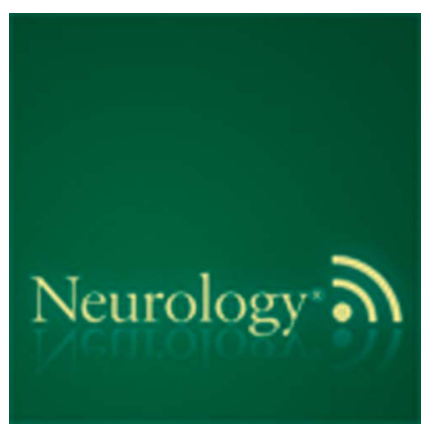

Evidence-based guideline update: Vagus nerve stimulation for the treatment of epilepsy (See p. 1453)

This podcast begins and closes with Dr. Robert Gross, Editor-inChief, briefly discussing highlighted articles from the October 15, 2013, issue of Neurology. In the second segment, Dr. Antel Patel talks with Dr. George Morris about the AAN guideline update on vagus nerve stimulation for the treatment of epilepsy. Dr. Adam Numis then reads the e-Pearl of the week about vita$\min \mathrm{B}_{6}$ and epilepsy. In the next part of the podcast, Dr. Mark Keegan focuses his interview with Dr. Robert Fox about dimethylfumarate for multiple sclerosis. Disclosures can be found at www.neurology.org.

At www.neurology.org, click on "RSS" in the Neurology Podcast box to listen to the most recent podcast and subscribe to the RSS feed.

CME Opportunity: Listen to this week's Neurology Podcast and earn 0.5 AMA PRA Category 1 CME Credits ${ }^{\mathrm{TM}}$ by answering the multiple-choice questions in the online Podcast quiz. 